\title{
Revelation and Physicalism
}

\author{
Kelly Trogdon, Virginia Tech
}

Abstract: According to experiential revelation, phenomenal concepts reveal the nature of the phenomenal properties they refer to. Some see experiential revelation as posing a direct challenge to physicalism. The basic idea is this: given experiential revelation, were phenomenal properties physical/functional in nature they would be presented as such when you think of them under phenomenal concepts, but phenomenal concepts don't present their referents in this way. I argue that, while this argument on a plausible reconstruction fails, the thesis of experiential revelation nevertheless indirectly challenges physicalism. In particular, it potentially undermines the so-called phenomenal concept strategy, a key defense maneuver of the physicalist for responding to dualist arguments concerning experience. The moral is that issues concerning revelation do indeed pose a problem for physicalism, but not for the reasons you might think.

\section{Introduction}

According to experiential revelation, phenomenal concepts reveal the nature of the phenomenal properties they refer to. Experiential revelation figures prominently in the literature on phenomenal concepts. Chalmers, for example, claims that a phenomenal concept is a concept that picks out its referent "in terms of its intrinsic nature" (2003, 225). According to Schroer, phenomenal concepts provide a "characterization of the intrinsic nature of their referents" (2010, 505). According to Nida-Rümelin, we grasp phenomenal properties via phenomenal concepts and "to grasp a property is to understand what having that property essentially consists in" (2007, 307). Horgan and Teinson claim that, when you think of a phenomenal property under a phenomenal concept, you conceive of that property "directly, as it is in itself" (2001, 311). Goff claims that a phenomenal concept is a concept that "reveals the nature of its referent" $(2011,194)$. The core idea here is that a phenomenal concept puts you, as it were, into cognitive contact with the nature of the property it refers to.

How can we sharpen up the idea that phenomenal concepts reveal the nature of the phenomenal properties they refer to? I begin with the notion of essence. One property has another essentially just in case part of what it is to be the 
former is that it has the latter. Here's a true if not very exciting claim: if the employees of a trucking company are picketing outside their workplace then there is a labor strike in virtue of this fact. So consider the following property: being such that if there are employees behaving in thus-and-so way then there is a strike in virtue of this fact. ${ }^{1}$ Call this property strike*. The property strike has strike* essentially-part of what it is to be the property strike is that if there are employees behaving in thus-and-so way then there is a strike in virtue of this fact. $^{2}$

Now I turn to the notion of characterization. Intuitively, concepts characterize or represent their referents as being a certain way. I take it that how a concept characterizes its referent is a dimension of that concept's meaning. Characterization so understood likely requires a two-factor conception of meaning according to which content and reference are distinct. For consider the referentialist view according to which the content and referent of a concept are the same. It seems that the referentialist would make one of two claims about characterization: either concepts just don't characterize what they refer to (they only refer), or they do, provided that we understand the notion of characterization so that it isn't a dimension of meaning. The referentialist might claim, for example, that concepts differ in the characterizations they provide of their referents just in case they're different Mentalese formulae (Fodor 2008, Ch. 3). In either case, characterization isn't a dimension of meaning.

Consider, by contrast, conceptual role semantics, here understood as the twofactor view according to which the content facts are (fully) grounded in facts about the conceptual roles of concepts (Block 1986 and Peacocke 1992).

1 The 'thus-and-so' locution here is a placeholder either for a demonstrative or a demonstrative-free description-I leave the matter open. I treat future instances of the locution in the same way.

2 Strike* is an indiscriminately necessary property-every entity has the property necessarily. But, while Socrates, for example, has strike* necessarily, he doesn't have it essentially-it's not part of what it is to be Socrates that if there are employees behaving in thus-and-so way then there is a strike in virtue of this fact. While something has a property necessarily provided that it has that property essentially, from the fact that something has a property necessarily it doesn't follow that it has that property essentially (Fine 1994). 
Focusing on inferences, in this case we can say that a concept $C$ characterizes its referent $\mathrm{P}$ in terms of $\mathrm{Q}$ (equivalently: $\mathrm{C}$ represents $\mathrm{P}$ as having $\mathrm{Q}$ ) just in case dispositions to make inferences to the effect that $\mathrm{P}$ has $\mathrm{Q}$ are part of C's conceptual role (equivalently: inferences to the effect that $\mathrm{P}$ has $\mathrm{Q}$ are meaning-constitutive with respect to $\mathrm{C}$ ). For the purposes of this paper I assume that conceptual role semantics is true and I work with this conception of characterization.

Finally, the notion of essential characterization: $\mathrm{C}$ provides an essential characterization of its referent $\mathrm{P}$ just in case there is some Q such that $\mathrm{P}$ has $\mathrm{Q}$ essentially and $\mathrm{C}$ characterizes $\mathrm{P}$ in terms of Q. Consider, for example, the concept STRIKE and its referent strike. Assuming that STRIKE characterizes strike in terms of strike* and the former has the latter essentially, it follows that STRIKE provides an essential characterization of its referent. Suppose that strike also has the property being the most discussed property in labor disputes, and there is a concept that refers to strike and characterizes it in terms of this property. Strike doesn't have this property essentially. And suppose that any other property in terms of which this concept characterizes strike is a non-essential property of strike. It follows that this concept provides a non-essential characterization of its referent.

Putting these ideas together, we can understand experiential revelation as the thesis that phenomenal concepts provide essential characterizations of the phenomenal properties they refer to. Why accept the thesis of experiential revelation so understood? In clarifying the notions of a phenomenal property and a phenomenal concept it will become clear that the thesis so understood is plausible.

To begin, what is a phenomenal property? Phenomenal properties are properties that type mental states by what it's like to have them. As Bill looks at a red tomato he has a particular visual experience. Had he looked at a green apple instead he would have had a visual experience of a different type. While the first experience falls under various types that the second doesn't and vice versa, the relevant difference between them is that they're different in terms of what it's like to have them-in the first case Bill has a state that instantiates phenomenal red but not phenomenal green, while in the second he has a state that instantiates the latter but not the former. Consider the property being such that if a person has a phenomenally red state then the person feels like thus-and-so in virtue of this 
fact. Call this property phenomenal red*. Phenomenal red has phenomenal red* essentially_ part of what it is to be the property phenomenal red is that if a person has a phenomenally red state then the person feels like thus-and-so in virtue of this fact. This is a natural way of clarifying the intuitive idea that phenomenal red is essentially tied to feelings of a particular sort. The same applies to phenomenal green and other phenomenal properties. ${ }^{3}$

What is a phenomenal concept? On a first pass, a phenomenal concept is a concept that refers to a phenomenal property and presents its referent in terms of how subjects feel when they have states with that property. We can clarify this initial take on phenomenal concepts with the notion of characterization. Suppose that PHENOMENAL RED is a phenomenal concept, one that refers to phenomenal red. Inferences to the effect that phenomenal red has phenomenal red* are meaning-constitutive with respect to PHENOMENAL RED. In other words, PHENOMENAL RED characterizes phenomenal red in terms of phenomenal red*. Generalizing, a more perspicuous take on phenomenal concepts is this: a phenomenal concept is a concept that refers to a phenomenal property and characterizes its referent in terms of experiential properties like phenomenal red*.

Given that phenomenal red has phenomenal red* essentially and PHENOMENAL RED characterizes the former in terms of the latter, it follows that PHENOMENAL RED provides an essential characterization of its referent. The same applies to other phenomenal concepts. Suppose that phenomenal red also has the property being the least discussed property in labor disputes, and there is a concept that refers to phenomenal red and characterizes it in terms of this property. Phenomenal red has this property non-essentially, and this property isn't an experiential property like phenomenal red*. Further, suppose that any other property in terms of which the concept characterizes phenomenal red is neither an experiential property nor an essential property of phenomenal red. It follows that the concept is neither a phenomenal concept nor one that provides an essential characterization of its referent.

If what I've said above about phenomenal properties and phenomenal concepts is correct then experiential revelation is true. But why care whether

${ }_{3}$ Phenomenal red and the property feeling like thus-and-so are distinct, as the former is a property of states while the latter is a property of subjects with states. The same distinction applies to other phenomenal properties. 
the thesis is true? Well, according to a posteriori physicalism, (i) any mental property instantiated in the actual world is identical to some physical/functional property, and (ii) the fact that this is so isn't knowable a priori, as phenomenal concepts are inferentially isolated from physical/functional concepts. The latter include neurophysiological concepts such as C-FIBER FIRING and causal role concepts such as THE PROPERTY OF HAVING SOME PROPERTY OR OTHER THAT PLAYS THUS-AND-SO CAUSAL ROLE. (Henceforth, when I speak of physicalism, understand this to be a posteriori physicalism in particular.) Horgan and Tienson (2001) as well as Goff (2011, 2015) argue that experiential revelation poses a direct challenge to physicalism, offering versions of what I call the revelation argument.

In the next section I set out a plausible reconstruction of the revelation argument that proceeds upon the notion of essential characterization. I show that the argument is unpersuasive on this reconstruction. In the following section I argue that experiential revelation nevertheless poses an indirect challenge to physicalism, as it potentially undermines the phenomenal concept strategy, a key defensive maneuver of the physicalist for responding to dualist arguments concerning experience. The moral is that issues concerning revelation do indeed pose a problem for physicalism, but not for the reasons you might think.

\section{Revelation as a direct challenge to physicalism}

\subsection{The revelation argument}

It's a familiar idea that some concepts present their referents as being physical/functional in nature. We can sharpen this idea as follows: a concept provides a physical/functional characterization of its referent $\mathrm{P}$ just in case inferences to the effect that $\mathrm{P}$ is a physical/functional property are meaningconstitutive with respect to that concept. Cast in terms of the notions of essential and physical/functional characterization, the revelation argument goes like this:

(1) PHENOMENAL RED provides an essential characterization of its referent, phenomenal red.

(2) PHENOMENAL RED doesn't provide a physical/functional characterization of phenomenal red. 
(3) If PHENOMENAL RED provides an essential but not a physical/functional characterization of phenomenal red then this property isn't a physical/functional property.

(4) Hence, phenomenal red isn't a physical/functional property.

The first premise of the argument is a special case of experiential revelation. The second premise is common ground between the dualist and physicalistboth agree that phenomenal concepts don't present their referents as being physical/functional in nature. And the rationale for the third premise- the linking premise - is straightforward. Suppose for the moment that phenomenal red is a physical/functional property. If the nature of this property is revealed to you when you think of it under PHENOMENAL RED then the property's status as a physical/functional property is likewise revealed to you when you do so. For how could the fact that it's a physical/functional property elude you when you grasp what it is to be phenomenal red? After all, if phenomenal red has the property being a physical/functional property, it has this property essentially. Hence, if phenomenal red is a physical/functional property then it's not the case that PHENOMENAL RED provides an essential but not a physical/functional characterization of its referent. ${ }^{4}$

What should we make of this argument? Some target the first premise, as they reject experiential revelation. Balog claims that the felt aspect of tokening PHENOMENAL RED and what it feels like to have a state with phenomenal red are the same, and this fact "produces the sense that [you have] direct insight into the nature of the experience" when you token the concept. But in reality PHENOMENAL RED "does not reveal anything about the metaphysical nature of phenomenality” (2012a, 30-1). McLaughlin rejects experiential revelation as well-he claims that phenomenal concepts "do not conceptually reveal anything about the essential nature of phenomenal properties" (2001, 324). And Veillet (forthcoming) claims that the physicalist should reject experiential revelation, as it's obviously incompatible with physicalism.

\footnotetext{
${ }^{4}$ Here's how Horgan and Tienson put the idea: “... if indeed phenomenal properties, when conceived under phenomenal concepts, not only are conceived otherwise than as physical-functional properties but also are conceived as they are in themselves, then surely phenomenal properties must be otherwise than physical-functional properties" (2001, 311).
} 
Experiential revelation, however, is prima facie plausible, and this motivates exploration of distinct responses to the revelation argument. In what follows I raise two problems for the argument while granting that experiential revelation (properly understood) is true. Both concern different readings of the term 'essential characterization'. The first concerns the distinction between partial and full essential characterization; the second concerns the distinction between what I call partially expressive full essential characterization and fully expressive full essential characterization.

\subsection{Problem 1: partial vs. full essential characterization}

When I introduced the notion of essential characterization I introduced the notion of partial essential characterization in particular. By contrast, $C$ provides a full essential characterization of its referent $\mathrm{P}$ just in case, for any property $\mathrm{Q}$, if $\mathrm{P}$ has $\mathrm{Q}$ essentially then $\mathrm{C}$ characterizes $\mathrm{P}$ in terms of $\mathrm{Q}$. It's necessary that if a concept provides a full essential characterization of its referent then it provides a partial essential characterization of its referent, but not vice versa. (I assume that any property has at least one property essentially.)

Here is the first problem with the revelation argument: (i) while the first premise is plausible when 'essential characterization' is read as 'partial essential characterization', there is no apparent reason to think it's true when 'essential characterization' is read as 'full essential characterization'; and (ii) while the linking premise may be true when 'essential characterization' is read as 'full essential characterization', it's implausible when 'essential characterization' is read as 'partial essential characterization'.

Consider the first premise: phenomenal red has phenomenal red* essentially and PHENOMENAL RED characterizes the former in terms of the latter. But note that, for all I've said, phenomenal red has some other property essentially as well, and it's not the case that PHENOMENAL RED characterizes its referent in terms of this property. So, while PHENOMENAL RED provides a partial essential characterization of its referent, nothing I've said so far suggests that it provides a full essential characterization. Moral: while the first premise is plausible when 'essential characterization' is read as 'partial essential characterization', there is 
no evident reason to think that it's true when 'essential characterization' is read as 'full essential characterization'.

Now I turn to the linking premise. Let's grant for the sake of argument that the linking premise is plausible when 'essential characterization' is read as 'full essential characterization'. (I return to this assumption later.) What if 'essential characterization' is read instead as 'partial essential characterization'? Well, suppose that phenomenal red is a physical/functional property. It follows that phenomenal red has being a physical/functional property essentially. The claim that PHENOMENAL RED provides a partial essential characterization of phenomenal red is compatible with the concept not characterizing its referent in terms of being a physical/functional property or any other property the possession of which renders phenomenal red a physical/ functional property (e.g. being the property of having some property or other that plays thus-and-so causal role). Hence, phenomenal red being a physical/functional property is compatible with PHENOMENAL RED providing a partial essential characterization and not a physical/functional characterization of its referent. Moral: while the linking premise may be plausible when 'essential characterization' is read as 'full essential characterization', there are no apparent grounds for accepting it when 'essential characterization' is read as 'partial essential characterization'.

${ }^{5}$ Chalmers (2003) is naturally read as claiming that phenomenal concepts provide full essential characterizations of their referents. I read Horgan and Tienson (2001) and Goff $(2011,2015)$ as staying neutral on whether these characterizations are full or merely partial, and Nida-Rümelin (2007) and Schroer (2010) as claiming that they're merely partial in nature.

${ }^{6}$ Arnauld offers a similar critique of Descartes' real distinction argument. Arnauld imagines that, while you might clearly and distinctively understand that a particular triangle is right-angled, you might wrongly think that the Pythagorean theorem doesn't apply to it. In this case you would wrongly think that the applicability of the theorem falls outside of the triangle's nature. By analogy, while Descartes clearly and distinctively understands his nature to be something that thinks, he might be wrong in thinking that nothing else belongs to his nature apart from the fact he's a thinking thing - perhaps the fact that he's an extended thing also belongs to his nature. Thanks to Martin Hann for pointing me to Arnauld's discussion. 


\subsection{Problem 2: fully expressive vs. partially expressive full essential characterization}

Elpidorou (forthcoming) also argues that the physicalist should respond to the revelation argument by claiming that phenomenal concepts reveal only part of the nature of their referents. (His argument, however, has significant differences from mine-I return to it in the next section.) As I read Elpidorou, he grants that if PHENOMENAL RED reveals all of what it is to be phenomenal red then there is indeed a real problem for the physicalist. By contrast, I think that the revelation argument fails even in this case.

Belief is opaque in nature. Suppose that you believe that Superman can fly. In this case it doesn't follow that you believe that Clark Kent can fly. Similarly, suppose that the predicates 'Bob's actual favorite property' and 'being blue' rigidly designate the same property. ${ }^{7}$ Supposing that you believe that Bill is blue, it doesn't follow that you believe that Bill has Bob's actual favorite property. What's true of belief is true of concepts. Suppose that $C$ characterizes $\mathrm{P}$ in terms of $\mathrm{Q}$. If ' $\mathrm{Q}$ ' and ' $\mathrm{R}$ ' rigidly designate the same property, it doesn't follow that $C$ characterizes $P$ in terms of $R$. In other words, if inferences to the effect that $\mathrm{P}$ has $\mathrm{Q}$ are meaning-constitutive with respect to $C$, if ' $R$ ' in addition to ' $Q$ ' rigidly designates $Q$, it doesn't follow that inferences to the effect that $\mathrm{P}$ has $\mathrm{R}$ are also meaning-constitutive with respect to $\mathrm{C}$.

With this in mind, consider the distinction between partially expressive and fully expressive full essential characterization. $\mathrm{C}$ provides a partially expressive full essential characterization of its referent $\mathrm{P}$ just in case, for any $\mathrm{Q}$ that $\mathrm{P}$ has essentially, there is some predicate ' $R$ ' that rigidly designates $Q$ such that $C$ characterizes $\mathrm{P}$ in terms of $\mathrm{R}$. (Equivalently: $\mathrm{C}$ provides such a characterization of $\mathrm{P}$ just in case, for any $\mathrm{Q}$ that $\mathrm{P}$ has essentially, there is some predicate ' $\mathrm{R}$ ' that rigidly designates $\mathrm{Q}$ such that inferences to the effect that $\mathrm{P}$ has $\mathrm{R}$ are meaning-constitutive with respect to $\mathrm{C}$.) $\mathrm{C}$ provides a fully expressive full

\footnotetext{
7 This isn't to say, however, that the predicate 'being Bob's actual favorite property' and 'being blue' rigidly designate the same property — the property designated by the former is a second-order property while the property designated by the latter isn't.
} 
essential characterization of its referent $\mathrm{P}$ just in case, for any $\mathrm{Q}$ that $\mathrm{P}$ has essentially and any predicate ' $R$ ' that rigidly designates $Q, C$ characterizes $P$ in terms of R. (Equivalently: C provides such a characterization of $\mathrm{P}$ just in case, for any $\mathrm{Q}$ that $\mathrm{P}$ has essentially and any predicate ' $\mathrm{R}$ ' that rigidly designates $\mathrm{Q}$, inferences to the effect that $\mathrm{P}$ has $\mathrm{R}$ are meaning-constitutive with respect to C.) It's necessary that if $\mathrm{C}$ provides a fully expressive full essential characterization of $\mathrm{P}$ then $\mathrm{C}$ provides a partially expressive full essential characterization of $\mathrm{P}$, but not vice versa.

Returning to the revelation argument, grant for the sake of argument that phenomenal concepts provide full essential characterizations of their referents. But talk of full essential characterization, as I have just indicated, can be taken in two ways. By attending to this ambiguity another problem for the revelation argument comes into focus: (i) while the first premise may be true when 'full essential characterization' is read as 'partially expressive full essential characterization', it's false when 'full essential characterization' is read as 'fully expressive full essential characterization'; and (ii) while the linking premise is true when 'full essential characterization' is read as 'fully expressive full essential characterization', there is no apparent reason to think that it's true when 'full essential characterization' is read as 'partially expressive full essential characterization'.

I begin with the first premise. PHENOMENAL RED doesn't provide a fully expressive full essential characterization of its referent because no concept (well, none that we possess, anyway) characterizes its referent in this way. Such a concept would not only characterize its referent in terms of all of its essential properties, but it would do so, as it were, under every possible guise for these properties. Suppose, for example, that 'Bill's actual favorite property' rigidly designates phenomenal red*. If PHENOMENAL RED provides a fully expressive full essential characterization of its referent then PHENOMENAL RED represents phenomenal red as having Bill's actual favorite property, but clearly it doesn't. That PHENOMENAL RED doesn't provide a fully expressive full essential characterization of its referent, however, is compatible with it providing a partially expressive full essential characterization of its referent. Moral: while the first premise is false when 'full essential characterization' is read as 'fully expressive full essential characterization', it may be true when 'full essential characterization' is read as 'partially expressive full essential characterization'. 
Now I turn to the linking premise. If PHENOMENAL RED provides a fully expressive full essential characterization but not a physical/functional characterization of its referent, it follows that phenomenal red isn't a physical/functional property. But if PHENOMENAL RED instead provides a partially expressive full essential characterization but not a physical/functional characterization of its referent it doesn't follow that phenomenal red isn't a physical/functional property. Suppose that phenomenal red is a functional property, the property of having some property or other that plays thus-and-so causal role. In this case phenomenal red has the property being the property of having some property or other that plays thus-and-so causal role essentially. Call this property causal role. Now consider the following two claims: (i) there is some nonphysical/functional predicate ' $\mathrm{N}$ ' such that ' $\mathrm{N}$ ' rigidly designates causal role and PHENOMENAL RED characterizes phenomenal red in terms of $\mathrm{N}$; and (ii) there is no physical/functional predicate ' $\mathrm{P} / \mathrm{F}$ ' such that ' $\mathrm{P} / \mathrm{F}$ ' rigidly designates causal role and PHENOMENAL RED characterizes phenomenal red in terms of P/F. (i) and (ii) are compatible with PHENOMENAL RED providing a partially expressive full essential characterization of its referent. Now consider the following two more general claims: (iii) for any property $\mathrm{P}$ that phenomenal red has essentially, there is some non-physical/functional predicate ' $\mathrm{N}$ ' such that ' $\mathrm{N}$ ' rigidly designates $\mathrm{P}$ and PHENOMENAL RED characterizes phenomenal red in terms of $\mathrm{N}$; and (iv) for any property $\mathrm{P}$ that phenomenal red has essentially, there is no physical/functional predicate ' $\mathrm{P} / \mathrm{F}$ ' such that ' $\mathrm{P} / \mathrm{F}$ ' rigidly designates $\mathrm{P}$ and PHENOMENAL RED characterizes phenomenal red in terms of $\mathrm{P} / \mathrm{F}$. If (iii) and (iv) are correct then PHENOMENAL RED provides a partially expressive full essential but not a physical/functional characterization of its referent, despite the fact that phenomenal red is a functional property. Moral: while the linking premise is plausible when 'full essential characterization' is read as 'fully expressive full essential characterization', this plausibility isn't preserved when 'full essential characterization' is read as 'partially expressive full essential characterization'.

\subsection{Further thoughts on the revelation argument}

Here are some further thoughts on the revelation argument, including how my objections compare to previous responses. Let's begin by returning to the first problem I raised for the argument. According to Goff (2011), appealing to the idea that phenomenal concepts reveal only part of the nature of their referents doesn't help the physicalist here. He reasons as follows (here I use my terminology rather than his). Suppose that PHENOMENAL RED provides a mere 
partial essential characterization of its referent. In this case, phenomenal red has some property P essentially, and PHENOMENAL RED characterizes phenomenal red in terms of $\mathrm{P}$. Now, if phenomenal red is a physical/functional property, any property it has essentially is itself a physical/functional property. Hence, if phenomenal red is a physical/functional property it follows that $\mathrm{P}$ is a physical/functional property. Goff's key claim is this: if $\mathrm{P}$ is a physical/functional property and PHENOMENAL RED characterizes phenomenal red in terms of $\mathrm{P}$, it follows that PHENOMENAL RED provides a physical/functional characterization of its referent. So, given that PHENOMENAL RED characterizes phenomenal red in terms of P but it doesn't provide a physical/functional characterization of its referent, it must be that phenomenal red isn't a physical/functional property.

Given the discussion in the previous section, however, we can see that Goff's key claim is insufficiently motivated. Since PHENOMENAL RED doesn't characterize phenomenal red in terms of $\mathrm{P}$ under all of P's guises, the fact that PHENOMENAL RED doesn't provide a physical/functional characterization of its referent is compatible with the idea that PHENOMENAL RED characterizes phenomenal red in terms of $\mathrm{P}$ where $\mathrm{P}$ is a physical/functional property.

Let's now return to Elpidorou (forthcoming). Elpidorou's discussion proceeds upon the distinction between physical/functional properties in the restricted sense and broad sense. Roughly speaking, a property is physical/functional in the restricted sense just in case it's a physical/functional property that if instantiated isn't instantiated in virtue of other properties; and a property is physical/functional in the broad sense just in case if it's instantiated then it's instantiated ultimately in virtue of physical/functional properties in the restricted sense. Elpidorou's proposal is that "...introspection reveals that phenomenal states are physical (in a broad sense) without at the same time revealing that phenomenal states are [instantiated in virtue of] physical (in the restricted sense) properties" (forthcoming, 18). I interpret this proposal as challenging something like the second premise of the revelation argument on my reconstruction (Elpidorou works with a slightly different reconstruction of the argument). He contrasts his proposal with the following view: introspection reveals that phenomenal states have certain properties essentially that turn out to be physical/functional in the broad sense, but introspection doesn't reveal that these properties are in fact physical/functional in the broad 
sense. My property guise proposal is essentially a special case of the latter proposal.

Returning to PHENOMENAL RED, let's call inferences to the effect that phenomenal red has some property that's instantiated ultimately in virtue of physical/functional properties in the restricted sense broadly physical inferences. Elpidorou's proposal is specifically that PHENOMENAL RED reveals that phenomenal red has a physical/functional property in the broad sense in a way that doesn't require that broadly physical inferences be meaning-constitutive with respect to PHENOMENAL RED. Now, I understand talk of concepts revealing things about their referents in terms of concepts characterizing their referents in particular ways, and I have a particular take on what characterization comes to, as I've previously indicated. Note, however, that Elpidorou doesn't have my package of views in mind in his discussion-on my view if PHENOMENAL RED represents its referent as having a physical/functional property in the broad sense, this is because certain broadly physical inferences are meaning-constitutive with respect to PHENOMENAL RED. I'm not confident that there is a good alternative way of understanding revealment talk in this context. At any rate, as Elpidorou is making importantly different assumptions about what it is for a concept to reveal something about its referent, I'll set his take on the revelation argument to the side.

Diaz-Leon (2014) also argues that Goff's $(2011,2015)$ version of the revelation argument fails, focusing on the idea that there are different ways to know part of the nature of a property. I see Diaz-Leon's discussion as leading to the following objection to the linking premise in the revelation argument, though the details here go beyond what she explicitly commits herself to.

The objection has five components. First, let $<\mathrm{p}>$ (the proposition that $\mathrm{p}$ ) represent the fact that phenomenal red has phenomenal red* essentially, where $<\mathrm{p}>$ is structured in part by PHENOMENAL RED. Second, suppose you possess some concept $\mathrm{C}$ that refers to physical/functional property $\mathrm{P}_{1}$. Third, suppose that $\mathrm{P}_{1}$ has physical/functional property $\mathrm{P}_{2}$ essentially. Fourth, suppose that $\mathrm{C}$ characterizes $\mathrm{P}_{1}$ in terms of $\mathrm{P} / \mathrm{F}$, where ' $\mathrm{P} / \mathrm{F}$ ' is a physical/functional predicate that rigidly designates $\mathrm{P}_{2}$. Fifth, let $<\mathrm{q}>$ represent the fact that $\mathrm{P}_{1}$ has $\mathrm{P}_{2}$ essentially, where $<\mathrm{q}>$ is structured in part by $\mathrm{C}$. 
With these suppositions in place, if you know $<$ p $>$ and $<\mathrm{q}>$ then you know part of the nature of phenomenal red and part of the nature of $\mathrm{P}_{1}{ }^{8}$ Consistent with this, however, is the idea that phenomenal red and $\mathrm{P}_{1}$ are the same property, as are phenomenal red* and $\mathrm{P}_{2}$. In this case if you know $<\mathrm{p}>$ and $\langle\mathrm{q}\rangle$ then you know part of the nature of a property in different ways, and you know, as it were, the same part of the nature of that property in different ways. One of these ways of knowing the nature of the property involves a concept that provides a physical/functional characterization of its referent while the other doesn't.

This critique of the revelation argument is compatible with and quite similar to the second problem I raised for the argument. There are two important differences, however. First, my critique is more direct and thus appeals to fewer contentious assumptions. In particular, it makes no assumptions about what we know, and it requires neither a neo-Fregean conception of propositions (a conception of propositions according to which concepts are constituents of propositions), nor the idea that propositions represent facts. Second, the proposal under consideration works with the idea that our grasp of the nature of phenomenal properties is partial in nature. As with Elpidorou's proposal, it may be that an assumption working in the background of Diaz-Leon's discussion is that if PHENOMENAL RED reveals all of what it is to be phenomenal red then there is a real problem for the physicalist. My response to the revelation argument highlights the fact that even if phenomenal concepts represent their referents in this way the revelation argument nevertheless fails.

${ }^{8}$ Diaz-Leon would add a caveat: knowing $<\mathrm{p}>$ counts as knowing part of the nature phenomenal red provided that $<\mathrm{p}>$ plays a role in determining the content of PHENOMENAL RED. Why the caveat? Well, suppose that BILL'S ACTUAL FAVORITE PROPERTY refers to phenomenal red* and let $<\mathrm{p}^{*}>$ represent the fact that phenomenal red has phenomenal red* essentially, where this proposition is structured by PHENOMENAL RED and BILL'S ACTUAL FAVRORITE PROPERTY. Both Goff and Diaz-Leon seem to agree in this case that if you know $<\mathrm{p}^{*}>$ you don't thereby know part of the nature of phenomenal red. Diaz-Leon suggests that the reason why is that $\left\langle\mathrm{p}^{*}>\right.$ doesn't play a role in determining the content of PHENOMENAL RED for you. The same considerations apply, mutatis mutandis, to $<\mathrm{q}>$ and knowing part of the nature of $\mathrm{P}_{1}$. This proposal strikes me as plausible, but I gloss over it above. 


\section{Revelation as an indirect challenge to physicalism}

Consider the notion of partially expressive partial essential characterization: $\mathrm{C}$ provides a partially expressive partial essential characterization of its referent $\mathrm{P}$ just in case there is some Q that P has essentially and some predicate ' $R$ ' that rigidly designates $\mathrm{Q}$ such that $\mathrm{C}$ characterizes $\mathrm{P}$ in terms of $\mathrm{R}$. Henceforth understand experiential revelation as the thesis that phenomenal concepts provide partially expressive partial essential characterizations of their referents. And from now on when I say that a concept provides an essential characterization of its referent I mean that it provides a partially expressive partial essential characterization of its referent in particular.

In the previous section I argued that the revelation argument on a plausible reconstruction fails for two different reasons. What now? In this section I argue that experiential revelation nevertheless poses an indirect challenge to physicalism. In particular, it potentially undermines the phenomenal concept strategy for defending physicalism against dualist arguments concerning experience.

\subsection{The phenomenal concept strategy}

What is the phenomenal concept strategy (PCS)? I see the strategy as having three parts. The first involves a concession of sorts to the dualist: zombies are conceivable, Mary learns something about her experiences as well as the experiences of others when she leaves her black and white room, there is an explanatory gap between the experiential truths and the physical/functional truths, and we have an intuition of distinctness with respect to the experiential and physical/functional. Let's say that these facts collectively comprise our epistemic/semantic situation with respect to experience.

The second part: we can explain our epistemic/semantic situation without appealing to the idea that phenomenal properties are non-physical/functional in nature. Moreover, in this context we needn't make any claim about the nature of phenomenal properties that is potentially problematic for physicalism, such as the claim that phenomenal properties are by their nature tied to particular feelings. So we needn't appeal to the idea that phenomenal red has phenomenal red* essentially and other phenomenal properties have corresponding properties essentially. Instead, we can explain our epistemic/semantic situation by appealing to the nature of phenomenal concepts, in particular their epistemic/semantic features. As Balog puts the 
idea, it is "not the nature of qualia but rather the nature of the concepts in terms of which we think about qualia" that PCS would have us explain our epistemic/semantic situation in terms of (Balog 2012b, 6). The overall idea is that with recourse to the special features of phenomenal concepts we can get the explanation we want-we needn't attribute anything special to phenomenal properties themselves. ${ }^{?}$

The third part: the epistemic/semantic features of phenomenal concepts aren't primitive-we can explain why phenomenal concepts have these features, again without appealing to the idea that phenomenal properties are nonphysical/functional in nature. Moreover, in this context we needn't make any claim about phenomenal properties or phenomenal concepts that is potentially problematic for physicalism, such as the claim that phenomenal red has phenomenal red* essentially or the claim that in tokening phenomenal concepts we stand in a primitive cognitive relation of acquaintance to phenomenal properties. ${ }^{10}$ The overall idea is that, while phenomenal concepts are special, we can explain their special features and there is no question about whether their specialness is compatible with physicalism. ${ }^{11}$

${ }^{9}$ See Balog 2009 for further discussion of this aspect of PCS.

${ }^{10}$ For a discussion of acquaintance understood as a primitive cognitive relation and how this relates to PCS, see Levine 2007.

${ }^{11}$ It's potentially misleading to speak of the phenomenal concept strategy, as there are different ways of understanding the strategy's goal as well as just what we're allowed to appeal to in trying to achieve the goal. A relatively unambitious conception of the goal is this: our job is just to explain why phenomenal and physical/functional concepts are conceptually isolated (Diaz-Leon 2010). A relatively ambitious conception is this: our job includes explaining not only what I call our epistemic/semantic situation but also why we have the experiential knowledge that we do (Chalmers 2007). A relatively restrictive take on what we're allowed to appeal to is this: we must work with topic-neutral characterizations of both phenomenal concepts and our epistemic/semantic situation, where a characterization is topic-neutral when it's free of experiential terminology (Chalmers 2007). A relatively unrestrictive take is this: we're allowed to appeal to the idea that phenomenal properties are by their nature connected to particular feelings. My discussion of PCS in this paper addresses a particular approach to the 
If PCS can be successfully implemented then challenges to physicalism such as the conceivability argument, the knowledge argument, and the explanatory gap argument lose much of their bite, for these objections proceed upon the idea that it's special features of phenomenal properties that are ultimately responsible for our epistemic/semantic situation. But our epistemic/semantic situation can be explained without appealing to special features of phenomenal properties given a successful implementation of PCS. Taking the explanatory gap between the experiential and the physical/functional as an example, in this case it's the specialness of phenomenal concepts rather than anything special about phenomenal properties that explains why there is this explanatory gap, and there is no new explanatory gap regarding the epistemic/semantic features of phenomenal concepts.

What are the epistemic/semantic features of phenomenal concepts relevant to implementing PCS? It would seem that the fact that phenomenal concepts provide essential characterizations of their referents is a relevant feature. Let's focus on the fact that we have an intuition of distinctness with respect to the experiential and the physical/functional. Suppose that the physicalist claims that phenomenal red is identical to physical/functional property P. Against this claim the dualist points out, among other things, that we have an intuition of distinctness with respect to phenomenal red and $\mathrm{P}$ - it strikes us that these properties are distinct. How might the physicalist explain away this intuition? Proposal: (i) PHENOMENAL RED provides an essential characterization of its referent; (ii) whatever physical/functional concept refers to $\mathrm{P}$, that concept provides a non-essential characterization of its referent; and (iii) the truth of (i) and (ii) predicts that it would seem to us that phenomenal red and $\mathrm{P}$ are distinct. ${ }^{12}$

strategy's goals and assumptions, one that falls in between the takes just described and hews closely to Balog's description of the strategy.

12 If a property has the property being a physical/functional property then the former has the latter essentially. And a concept provides a physical/functional characterization of its referent just in case it represents its referent as being a physical/functional property. Hence, if a concept provides a physical/functional characterization of its referent then it provides an essential characterization of its referent. Returning to (ii) from above, it follows that (ii) is true only if the physical/functional concept that refers to $\mathrm{P}$ doesn't provide a physical/functional characterization of its referent. (Note that the truth of (ii) is compatible with the idea that you're disposed to infer that the property in question is a 
It's this cognitive difference between the physical/functional concept and PHENOMENAL RED that explains why phenomenal red and P strike us as being distinct properties. ${ }^{13}$

While this proposal would need to be developed in much more detail to be successful, it seems promising as a beginning point for explaining the intuition of distinctness. Remember, however, that for any epistemic/semantic feature that we attribute to phenomenal concepts in explaining our epistemic/semantic situation, that feature needs to be explainable in a certain way. One of the conditions, as I've already noted, is this: the relevant explanation should appeal neither to a primitive cognitive relation of acquaintance nor the claim that phenomenal properties have experiential natures (i.e. that they're essentially tied to particular feelings). Call this the no controversy condition for explanations of the epistemic/semantic features of phenomenal concepts, as these claims are potentially problematic for physicalism. Supposing that PHENOMENAL RED provides an essential characterization of its referent, is there an explanation of why the concept has this feature that satisfies the no controversy condition?

It won't do to say that concepts as a matter of course provide essential characterizations of their referents, as they don't. Ordinary empirical concepts like WATER provide non-essential characterizations of their referents- the meaning-constitutive inferences with respect to WATER don't correspond to essential truths about being $\mathrm{H}_{2} \mathrm{O}$. It's true that mathematical concepts, by

physical/functional property — what's required is just that this disposition isn't meaning-constitutive with respect to the concept.) I assume that certain physical/functional concepts don't provide essential characterizations of their referents such as NEURON (more on NEURON shortly). If this is right then there are physical/functional concepts that don't provide physical/functional characterizations of their referents either.

13 This proposal is close to what Balog (2012b) proposes. While she rejects experiential revelation, she claims that phenomenal concepts seem to provide essential characterizations of their referents, and this appearance of essential characterization explains certain features of our epistemic/semantic situation. We will return to Balog on PCS shortly. For an attempt to implement PCS in a way that doesn't appeal to experiential revelation (either the idea that the thesis is true or that it merely seems true), see Papineau 2002, Ch. 6. 
contrast, do provide essential characterizations of their referents, but in this case we have - at least for a wide range of these concepts - a straightforward explanation of why this is so: such concepts were introduced by way of explicit definition. Similar considerations apply to response-dependent concepts like STRIKE. Phenomenal concepts, by contrast, aren't introduced by way of explicit definition. ${ }^{14}$

So what explains why experiential revelation is true? Well, there are the two claims that got things going in the first place: PHENOMENAL RED characterizes its referent in terms of phenomenal red*, and the referent of PHENOMENAL RED has phenomenal red* essentially. Call the former the characterization claim and the latter the essence claim. The characterization claim, as you will recall, is a natural way of clarifying the idea that phenomenal concepts present phenomenal properties in terms of how subjects feel when they have states with those properties. And the essence claim is a natural way of clarifying the idea that phenomenal properties are essentially related to particular feelings. The characterization and essence claims together explain why PHENOMENAL RED provides an essential rather than non-essential characterization of its referent. Call this explanation the simple explanation. The simple explanation, however, is off limits for the purposes of implementing PCS, as it appeals to the idea that phenomenal properties have an experiential nature. ${ }^{15}$

I think that the advocate of PCS is in a tough position here. Suppose that experiential revelation will play a role in any plausible implementation of PCS. Hence, a condition of adequacy for PCS is that there is an explanation of why phenomenal concepts provide essential characterizations of their referents that satisfies the no controversy condition. But how could there be such an explanation given that experiential revelation itself is motivated by the idea that phenomenal properties have experiential natures?

In the remainder of the paper I look at what I take to be the three most plausible approaches that the advocate of PCS might take in attempting to

14 Thanks to Martin Hann for helpful discussion here.

15 Why can't the advocate of PCS just say that the referent of PHENOMENAL RED has some essential property and the concept characterizes its referent as having this property? The problem is that this isn't an explanation of experiential revelation but merely a statement of it. 
provide an explanation of experiential revelation that satisfies the no controversy condition. The first appeals to the semantics of phenomenal concepts, the second their metasemantics, and the third their representational vehicles. I argue that none of these strategies are promising.

\subsection{The semantic approach}

According to the semantic approach the content of a phenomenal concept can provide the sort of explanation we're looking for. How might this work? You might claim, following Loar (1997), that phenomenal concepts provide demonstrative characterizations of their referents. A simple version of this view concerning PHENOMENAL RED is this: the concept represents its referent as being that property, and there is nothing else to the content of this concept. In other words, the only meaning-constitutive inferences with respect to PHENOMENAL RED are inferences to the effect that phenomenal red is that (demonstratively identified) property. Call this the thin demonstrative view about phenomenal concepts.

Suppose for the moment that the thin demonstrative view is correct. In this case, we're free to appeal to the content of PHENOMENAL RED in attempting to explain why this concept provides an essential rather than non-essential characterization of its referent, as our explanans in this case doesn't appeal to the nature of phenomenal red, experiential or otherwise. Still, there is an obvious problem: if all there is to the content of PHENOMENAL RED is that this concept represents what it refers to as being that property, PHENOMENAL RED doesn't provide an essential characterization of its referent. Since the point of the semantic approach is to explain (in a certain way) why PHENOMENAL RED provides such a characterization of its referent, the thin demonstrative view is of no help to us here.

Perhaps PHENOMENAL RED provides a more substantive demonstrative characterization of its referent than the one mentioned above. Call this the thick demonstrative view with respect to PHENOMENAL RED. You might claim, for example, that phenomenal concepts are hybrid concepts in that their content has both demonstrative and relational components. I'll consider two proposals along these lines.

First, you might claim that PHENOMENAL RED represents its referent as occupying a certain position in a quality space where the space itself is 
specified partly in demonstrative terms. Levin (2002) defends such a view, claiming that the content of phenomenal concepts contain "relational descriptions of quality spaces with 'slots' reserved for type-demonstratives that are normally acquired by having the experiences in question" (2007, 98). Suppose that each of the relational properties in virtue of which phenomenal red occupies its position in the relevant quality space is essential to phenomenal red. To fix ideas, suppose that the relational property of being more similar to phenomenal orange than phenomenal green is one such property. Suppose that PHENOMENAL RED characterizes its referent as being (among other things) a property that is more similar to this property (phenomenal orange) than that property (phenomenal green). It follows that PHENOMENAL RED provides an essential characterization of its referent.

An immediate problem is that the current proposal seems to violate the no controversy condition for PCS, as phenomenal red is phenomenally more similar to phenomenal orange than phenomenal green. Having a phenomenally red state is more similar to having a phenomenally orange state than a phenomenally green state in terms of what it's like to have these states. This comparison proceeds upon the idea that phenomenal red has an experiential nature so it's just the sort of consideration that we're not supposed to be appealing to in implementing PCS. ${ }^{16}$

Perhaps, however, there is a way of weakening the no controversy condition so that it on the one hand plausibly functions as a condition of adequacy for PCS (so it prevents us from appealing to features of phenomenal properties in an objectionable way) and on the other allows us to appeal to such essential relational properties of phenomenal properties in implementing the strategy. But I think that the proposal under consideration fails in any case.

The problem concerns the fact that the proposal appeals to extrinsic rather than intrinsic properties of phenomenal red. The relational property being more similar to

\footnotetext{
16 A related proposal: PHENOMENAL RED characterizes its referent as being (among other things) a property that stands in this relation (the relation of being phenomenally more similar to _ than __) to that property (phenomenal orange) and this property (phenomenal green). While the notion of phenomenal similarity in this case doesn't figure into the content of PHENOMENAL RED, the proposal nevertheless appeals to the idea that phenomenal red has an experiential nature.
} 
phenomenal orange than phenomenal green is an extrinsic property, as it's not the case that something has this property in virtue of how it is as opposed to how it's related to other things. While I haven't stressed this point, it seems that phenomenal concepts provide essential intrinsic characterizations of their referents. Returning to PHENOMENAL RED, Schroer puts the idea like this: "The phenomenal concept of phenomenal red, for example, does not just characterize its referent as more closely resembling phenomenal orange than phenomenal green; it also provides a relatively substantial characterization of how phenomenal red is on its own" $(2010,512)$. C provides an essential intrinsic characterization of its referent $\mathrm{P}$ just in case there is some $\mathrm{Q}$ such that $\mathrm{Q}$ is intrinsic, $\mathrm{P}$ has $\mathrm{Q}$ essentially, and $\mathrm{C}$ characterizes $\mathrm{P}$ in terms of Q. So, provided that the job of the advocate of PCS is to explain (in a particular way) why PHENOMENAL RED provides an essential intrinsic characterization of its referent in particular, the current proposal fails.

What about a version of the thick demonstrative view that appeals to intrinsic properties of phenomenal properties? Well, suppose that phenomenal red is a complex property, and it has determinates of certain quantitative properties as constituents. Suppose in particular that it has a determinate of phenomenal warmth - thus-and-so degree of phenomenal warmth — as a constituent. Hence, phenomenal red has the property of having thus-and-so degree of phenomenal warmth as a constituent. Call this property W. In this case, phenomenal red has W essentially_-part of what it is to be phenomenal red is to be a property with W. And $\mathrm{W}$ is plausibly regarded as an intrinsic property. Suppose that PHENOMENAL RED characterizes its referent as being (among other things) a property that has thus-and-so degree of that property (phenomenal warmth) as a constituent. It follows that PHENOMENAL RED provides an essential intrinsic characterization of its referent. Schroer defends a view similar to this version of the thick demonstrative view, stating, "....in addition to demonstratively identifying and separating [their component elements] from one another, our phenomenal concepts also provide an explicit description of how much of each (demonstratively identified) magnitude is possessed by the phenomenal color in question" $(2010,516)$.

Like the Levin inspired proposal, the Schroer inspired proposal appeals to the idea that phenomenal properties have an experiential nature. As such, it violates the no controversy condition for PCS. Let's suppose again, however, that there is a plausible way of weakening the no controversy condition so that 
such a proposal counts as a PCS-friendly potential explanation of experiential revelation. But the proposal seems to fail in any case.

The problem is that it doesn't generalize. That is to say, there are phenomenal concepts that the proposal doesn't apply to-it doesn't tell us why they provide essential rather than non-essential characterizations of their referents. Consider, for example, the determinate of phenomenal warmth — what I'll call determinate warmth — that ex hypothesi is a constituent of phenomenal red. Just as phenomenal red has phenomenal red* essentially, since determinate warmth is a phenomenal property it has a corresponding property essentially, being such that if a person has a state with determinate warmth then the person feels like thus-and-so in virtue of this fact. Call this property determinate warmth* ${ }^{*}$. We can think of determinate warmth in terms of how it feels to have a state with this property. That is to say, there is a phenomenal concept-DETERMINATE WARMTH— that refers to determinate warmth and characterizes its referent in terms of determinate warmth ${ }^{*}$. Hence, DETERMINATE WARMTH, like PHENOMENAL RED, provides an essential rather than non-essential characterization of its referent.

The problem is that determinate warmth doesn't itself have determinates of quantitative properties as components. More generally speaking, it seems that determinate warmth is simple-it seems to lack constituents altogether. Assuming that it is simple, we of course can't appeal to the idea that the referent of DETERMINATE WARMTH has some property as a constituent in explaining why it is that this concept provides an essential rather than non-essential characterization of its referent. ${ }^{17}$

\footnotetext{
${ }^{17}$ Schroer (2010) proposes that, while the characterizations that phenomenal concepts like PHENOMENAL RED provide of their referents are substantive, more specific phenomenal concepts like DETERMINATE WARMTH provide relatively thin characterizations of their referents. His version of the thick demonstrative view is intended to apply only to phenomenal concepts that provide substantive characterizations of their referents. Whether or not the characterization that DETERMINATE WARMTH provides of its referent is substantive in the relevant sense (see Veillet forthcoming for a discussion of just what the relevant sense of the term might be), what's important for our purposes is that DETERMINATE WARMTH provides an essential rather than non-essential characterization of its referent.
} 
I've only argued that three takes on a particular version of the semantic approach - a version that appeals to demonstratives - fail. But I think that the failure of the proposals I've considered points to a general problem with the semantic approach. The problem can be put as a dilemma. In advocating any version of the semantic approach, we'll either appeal to the idea that phenomenal concepts provide demonstrative characterizations of their referents or we won't. Suppose that we don't. In this case it's hard to see how we won't end up appealing to the idea that it's the specialness of phenomenal properties that explains our epistemic/semantic situation. This is what happens when we, for example, say that PHENOMENAL RED characterizes its referent in terms of phenomenal red*, where the latter is an essential property of the former.

Now suppose that we do appeal to the idea that phenomenal concepts provide demonstrative characterizations of their referents. In this case, I suspect that one of three things will happen, provided that we don't end up appealing to features of phenomenal properties in an objectionable way: either we won't successfully explain why any phenomenal concept provides an essential characterization of its referent (putting aside whether these characterizations appeal to intrinsic properties), we won't explain why any phenomenal concept provides an essential intrinsic as opposed to essential extrinsic characterization of its referent, or our proposal will be limited in an objectionable way. I conclude that the semantic approach isn't promising.

\subsection{The metasemantic approach}

You might think that, while the semantic approach isn't promising, a related approach might work. Regarding PHENOMENAL RED, the idea is this: identify a plausible condition that explains why this concept has the content it does-call this condition a metasemantic condition for PHENOMENAL RED - and then explain in a manner that comports with PCS why PHENOMENAL RED provides an essential (intrinsic) rather than non-essential characterization of its referent by appealing to this condition. Call this the metasemantic approach.

Suppose that we identify a plausible metasemantic condition for PHENOMENAL RED. Assuming that conceptual role semantics is true (and the inferential role of a concept is part of its content-determining conceptual role), the fact that inferences to the effect that phenomenal red has phenomenal red* are part of the inferential role of PHENOMENAL RED is a metasemantic condition for the 
concept. What we have here in effect is an explanation of the characterization claim I mentioned earlier, the claim that PHENOMENAL RED characterizes phenomenal red in terms of phenomenal red*. Recall that the characterization claim along with the essence claim make up the simple explanation for experiential revelation, where the essence claim is the claim that phenomenal red has phenomenal red* essentially.

So far, so good. But to cite an explanation of part of the explanans of an explanation for experiential revelation (the simple explanation) that's off the table for the purposes of implementing PCS obviously doesn't give us the explanation we're looking for. It's true that if we combine our metasemantic condition with the essence claim then we get an explanation of why PHENOMENAL RED provides an essential rather than non-essential characterization of its referent. But our explanans in this case (the metasemantic condition + the essence claim) is of no more help to us than the simple explanation (the characterization claim + the essence claim). This is because the essence claim itself has no place in an implementation of PCS for the reasons I provided above.

You might respond that we can explain why the essence claim itself is true and then combine this explanation with the metasemantic condition to get an explanation of experiential revelation that comports with PCS. But, putting aside the fact that we're not supposed to be appealing to the idea that phenomenal properties have experiential natures in implementing PCS, this proposal faces the following problem: it seems that for at least some phenomenal properties there is no explanation for why they have the natures that they do. Some essence facts-facts to the effect that thus-and-so property has thus-and-so property essentially_ just aren't apt for explanation (Dasgupta 2014). Consider, for example, the property boiling. Boiling has the property being such that if the molecules that compose a portion of water overcome the forces of attraction between them then the water boils in virtue of this fact. Supposing that the former has the latter essentially, it seems that there is no explanation of why this is so. It seems appropriate to say in this context that that's just how boiling is. The same holds for certain mental properties. Consider, for example, the fact that determinate warmth has determinate warmth* essentially. It seems that there is no explanation of why the former has the latter essentially. It seems appropriate to say in this context that that's just how determinate warmth is. 
Just to be clear, my claim isn't that the advocate of PCS must explain why phenomenal properties have the natures that they do. My claim is just that there is one way of trying to implement PCS — the metasemantic strategy- that does seem to require this. For at least some phenomenal properties, it seems that there isn't an explanation of why they have the natures that they do. While this on its own doesn't pose a direct challenge to physicalism (or put PCS itself in peril), it does count against the particular approach to implementing PCS discussed in this section.

I conclude that the metasemantic approach isn't promising. If neither the semantics nor the metasemantics of phenomenal concepts provide a PCSfriendly explanation of why PHENOMENAL RED provides an essential rather than non-essential characterization of its referent, what's left to appeal to?

\subsection{The vehicle approach}

You might argue that by appealing to features of the representational vehicles of phenomenal concepts we can provide the sort of explanation of experiential revelation necessary to successfully implement PCS. Call this the vehicle approach. How might the vehicle approach go?

Several proponents of phenomenal concepts claim that these concepts on canonical applications (self-attributions in which their referents are instantiated) are such that their tokens are constituted by tokens of their referents (Papineau 2002, Ch. 4; Chalmers 2003; Block 2006; Balog 2009, 2012a, 2012b; Gertler 2011, Ch. 4; 2012). Call this the referent constitution view of phenomenal concepts. Some claim that the referent constitution view is supported by introspection, citing the fact that we often seem to use the actual occurrence of an experience as a means of thinking about that experience.

Advocates of this view claim that it can help us explain why phenomenal concepts have some of their characteristic epistemic/semantic features. Balog, for example, writes, "From [the perspective of the referent constitution view], the puzzle that the explanatory gap presents is rather a trick the mind plays on itself as a result of the peculiar cognitive architecture involved in first-person phenomenal thought" (2012a, 31). So a natural thought is that we can explain in a manner that comports with PCS why phenomenal concepts provide essential characterizations of their referents by appealing to the idea that phenomenal concepts on canonical applications are such that their tokens are 
constituted by tokens of their referents. The motivation for the proposal, impressionistically put, is this: if the property that you're thinking about is physically present in the very concept you're using to think about that property, there's no cognitive distance between yourself and the object of your thought, and the property's special cognitive proximity or presence ensures that you grasp at least part of its nature.

What should we make of this version of the vehicle approach? It faces a fairly straightforward problem. The problem is that the reasoning outlined above leads to implausible claims when we consider different examples. All of our concepts (for the physicalist, anyway) are such that their tokens consist of neurons, so when you token NEURON- a concept that refers to being a neuronvarious tokens of being a neuron constitute this concept token. Part of what it is to be the property being a neuron is that if something has this property then it has a soma containing a cell nucleus. Given the reasoning above, NEURON characterizes its referent in terms of the property being such that if something is a neuron then it has a soma that contains a cell nucleus or some other property that being a neuron has essentially. But you can possess the concept NEURON and not be disposed to infer that something has a soma that contains a cell nucleus from the fact that it's a neuron. More generally speaking, NEURON resembles WATER in that it doesn't provide an essential characterization of its referent-the meaning-constitutive inferences with respect to NEURON don't correspond to essential truths about being a neuron.

What has gone wrong here? Levine (2007) warns us not to conflate the notions of physical presence and cognitive presence-whether a token of a property is physically present in a token of the concept you use to think about that property is one thing; whether the property is cognitively present in a special way when you use that concept to think about the property is another. Suppose that the fact that a phenomenal concept provides an essential rather than non-essential characterization of its referent is to be explained in terms of the special cognitive presence that the referents of these concepts enjoy when we token these concepts. In this case a plausible diagnosis of the failure of the proposal under discussion is this: it goes wrong in thinking that physical presence is sufficient for cognitive presence.

You might respond that, as a general matter, referential constitution doesn't secure essential characterization, but in the case of phenomenal concepts it 
does. But what is special about phenomenal concepts that accounts for this difference? Whatever you say here, presumably part of the story will be this: their referents are by their nature experiential, while the referent of NEURON, for example, "knows nothing" of experience. But to go in this direction seems to undermine PCS for the reasons discussed above.

I've only argued that a particular version of the vehicle approach to experiential revelation-one that appeals to the referent constitution viewfails. But the failure of this version of the vehicle approach points to a general problem with the approach. Again following Levine 2007, as a general matter the physical features of the tokens that implement a representational system aren't relevant to explaining its cognitive features - what's relevant to explaining its cognitive features is instead how these tokens relate to each other. So it seems misguided to think that we can explain why phenomenal concepts provide essential rather than non-essential characterizations of their referents by appealing to details concerning the representational vehicles of these concepts, whether these details concern how these vehicles are constituted or something else entirely.

Balog (2012a) and (2012b) offers the most detailed proposal concerning how appealing to the referent constitution view might help in implementing PCS. As I noted earlier, Balog rejects the idea that phenomenal concepts provide essential characterizations of their referents. I read Balog in particular as agreeing that PHENOMENAL RED characterizes phenomenal red in terms of phenomenal red* but denying that the former has the latter essentially. So Balog, of course, won't see the fact that referential constitution fails to explain experiential revelation in a PCS-friendly way as being a problem for the strategy. For Balog, an epistemic/semantic feature of phenomenal concepts that's relevant to implementing PCS instead concerns the fact that these concepts afford a "substantive grasp" of phenomenal properties-roughly, they seem to reveal the nature of the properties they refer to (2012b).

Do considerations involving cognitive architecture alone explain why phenomenal concepts have this feature? Balog concedes that they don't: "Neither a neurophysiological, nor a mere 'architectural' description of phenomenal concepts - e.g., that they are constituted by instances of the referent - can explain the key features of acquaintance and the substantial manner in which we think of phenomenal properties" (2012b, 15). If the fact 
that phenomenal concepts afford a substantive grasp of their referents is to be explained in terms of the special cognitive presence that phenomenal properties enjoy when we token these concepts, what Balog is in effect claiming here is that physical presence isn't sufficient for cognitive presence. So, while Balog and I disagree about just which epistemic/semantic features phenomenal concepts have, we seem to agree on this much: architectural considerations alone don't explain the epistemic/semantic features of phenomenal concepts relevant to implementing PCS.

\section{Conclusion}

I began the paper by reconstructing the revelation argument and arguing that the argument on this reconstruction is unpersuasive. The moral is that, so far as the revelation argument goes, there is no good reason to think that experiential revelation poses a challenge to physicalism. But, given experiential revelation, PCS can be effectively implemented only if there is a certain sort of explanation of why phenomenal concepts provide essential rather than nonessential characterizations of their referents. I argued that it's unclear that there is an explanation of the required sort.

I don't conclude that there isn't such an explanation to be had-that would be premature. Instead, I view experiential revelation as providing an indirect challenge to physicalism. The physicalist needs to respond to dualist arguments concerning experience and PCS is the most promising way she has of doing so, yet experiential revelation throws the strategy into doubt. That this is so lends further support to a general idea that Chalmers (2007), Levine (2007), and others have recently pursued: to take phenomenal concepts seriously is to accept that they have features that may preclude us from implementing PCS. ${ }^{18}$

\footnotetext{
18 I presented versions of this paper at the Philosophy of Mind Workshop at Virginia Tech (April 2015), the Southern Society for Philosophy and Psychology (April 2015), and (via Skype) the Victoria University of Wellington (May 2013). I wish to thank my audience members and particularly Martin Hann-my commentator at the SSPP_-for helpful feedback. Special thanks to Nathan Adams, Louise Antony, Stuart Brock, Sam Cowling, Tim Fuller, Brie Gertler, Philip Goff, Ben Jantzen, Daniel Kraemer, Joseph Levine, Tristram McPerhson, Gregory Novack, Ted Parent, Nathan Rockwood, and two anonymous referees for their help.
} 


\section{References}

Alter, T. and S. Walter (eds.) 2007. Phenomenal Concepts and Phenomenal Knowledge. Oxford UP.

Balog, K. 2009. "Phenomenal Concepts." In (eds.) McLaughlin et al, Oxford Handbook in the Philosophy of Mind. Oxford UP.

---- 2012a. “Acquaintance and the Mind-Body Problem.” In (eds.) Hill and Gozzano, New Perspectives on Type Identity: The Mental and the Physical. Cambridge UP.

---- 2012b. "In Defense of the Phenomenal Concept Strategy," Pbilosopby and Phenomenological Research 84: 1-23.

Block, N. 2007. “Max Black’s Objection To Mind-Body Identity.” In (eds.) Alter and Walter (2007).

---- 1986. "Advertisement for a Semantics for Psychology," Midwest Studies in Philosophy 10: 615-78.

Chalmers, D. 2003. "The Content and Epistemology of Phenomenal Belief." In (eds.) Smith and Jokic, Consciousness: New Essays. Oxford UP.

---- 2007. "Phenomenal Concepts and the Explanatory Gap.” In (eds.) Alter and Walter (2007).

Dasgupta, S. 2014. "The Possibility of Physicalism," Journal of Philosophy 111: 557592.

Diaz-Leon, E. 2010. "Can Phenomenal Concepts Explain the Epistemic Gap?" Mind 119: 933-951.

--- 2014. "Do A Posteriori Physicalists Get Our Phenomenal Concepts Wrong?" Ratio 27: 1-16.

Elpidorou, A. Forthcoming. "A Posteriori Physicalism and Introspection," Pacific Philosophical Quarterly.

Fine, K. 1994. "Essence and Modality," Philosophical Perspectives 8: 1-16.

Fodor, J. 2008. LOT 2. Oxford UP.

Gertler, B. 2011. Self-Knowledge. Routledge.

---- 2012. "Renewed Acquaintance." In (eds.) Smithies and Stoljar, Introspection and Consciousness. Oxford UP.

Gillett, C. and B. Loewer (eds.) 2001. Physicalism and Its Discontents. Cambridge UP. 
Goff, P. 2011. “A Posteriori Physicalists Get our Phenomenal Concepts Wrong," Australasian Journal of Philosophy 89: 191-209.

---- 2015. "Real Acquaintance and Physicalism.” In (eds.) P. Coates and S. Coleman, Phenomenal Qualities. Oxford UP.

Horgan, T. and J. Tienson. 2001. "Deconstructing New Wave Materialism.” In (eds.) Gillett and Loewer (2001).

Levin, J. 2002. "Is Conceptual Analysis Needed for the Reduction of Qualitative States?" Philosophy and Phenomenological Research 64: 571-591.

---- 2007. "What is a Phenomenal Concept?” In (eds.) Alter and Walter (2007).

Levine, J. 2007. "Phenomenal Concepts and the Materialist Constraint." In (eds.) Alter and Walter (2007).

Loar, B. 1997. "Phenomenal States." In (eds.) Block et al, The Nature of Consciousness. MIT Press.

McLaughlin, B. 2001. "In Defense of New Wave Materialism: A Response to Horgan and Tienson.” In (eds.) Gillett and Loewer (2001).

Nida-Rümelin, M. 2007. “Grasping Phenomenal Properties.” In (eds.) Alter and Walter (2007).

Papineau, D. 2002. Thinking About Consciousness. Oxford UP.

Peacocke, C. 1992. A Study of Concepts. MIT Press.

Schroer, R. 2010. "Where's the Beef? Phenomenal Concepts as Both Demonstrative and Substantial," The Australasian Journal of Philosophy 88: 505522.

Veillet, B. Forthcoming. "The Cognitive Significance of Phenomenal Knowledge," Philosophical Studies. 